\title{
Aus der Vortragsreihe Mein liebster Freud: «Erinnern, Wiederholen und Durcharbeiten" (1914g) oder der «Wolfsmann» zwischen Ödipus und Narziss, Tummelplatz und Krieg
}

\author{
Uorschla Guidon (Zürich)
}

Zusammenfassung: Der Artikel beruht aufeinem Vortrag, der in der Reihe "Mein liebster Freud» am 17. April 2014 am PSZ gehalten wurde. Die These lautet, dass Freud in der Abhandlung «Erinnern, Wiederholen und Durcharbeiten» (1914g) in seinem theoretischen Bezugsrahmen an der Neurose und am Modell des Traums orientiert ist, klinisch jedoch in den Bereich der nicht-neurotischen Störungen vorstösst. Das Agieren und Wiederholen in der Übertragung wird zum Äquivalent für das Erinnern von nicht (symbolisch) repräsentierten Erfahrungen, die auf diese Weise zur Darstellung gelangen. Es ergeben sich Anknüpfungspunkte für spätere Autoren wie Winnicott, Bion und Green, die sich mit der frühen Entwicklung befasst haben. Fragen der Entstehung des psychischen Raums und der Differenzierung von Innen und Aussen und dem Zwischen- und Übergangsraum werden erörtert.

Schlüsselwörter: Agieren, Wiederholungszwang, Grenzfälle, nicht repräsentierte Inhalte

\section{Einleitung}

Es gibt Sätze, die etwas so treffend auf den Punkt bringen, dass man sie nicht mehr vergisst. Bei einer Patientin, die ich seit vielen Jahren in Behandlung habe, halfen mir Freuds (1914g) Worte: «(...) der Analysierte erinnere überhaupt nichts von dem Vergessenen und Verdrängten, sondern er agiere es. Er reproduziert es nicht als Erinnerung, sondern als Tat, er wiederholt es, ohne natürlich zu wissen, dass er es wiederholt» (S. 129). Als die Patientin nach der X-ten Verspätung zögernd und wie schon zersetzt zur Sitzung erschien, bildete mein Gedanke, sie komme daher wie ein «geschlagener Hund» den Auftakt zu einer ersten, vorsichtigen Annäherung an ihre Geschichte, die von Schlägen, Entwertungen und Brüchen in der 
Kontinuität ihres Seins geprägt war. Die Patientin konnte ihre Geschichte nicht formulieren, sie trug sie gleichsam eingeprägt in ihre Körperhaltung zur Sitzung.

Die Feststellung Freuds, dass Patienten wiederholen und agieren, statt sich zu erinnern, ja dass sie nur auf diese Weise erinnern können, indem sie in der Übertragung agieren, was einmal war, halte ich für eine zentrale klinische Tatsache, ja für ein Kernstück der psychoanalytischen Praxis, gerade bei nicht-neurotischen Störungen.

Freud führt in der vorliegenden Arbeit den Wiederholungszwang ein. Anders als in "Jenseits des Lustprinzips» (1920g), wo er ihn mit dem Todestrieb in Verbindung bringt und von einem «dämonischen Zug» spricht (S. 20 und 36), erscheint der Wiederholungszwang hier unter der Perspektive der Aneignung von Erfahrung. Aber Freud skizziert bereits ein klinisches Bild, das ins «Jenseits» der Neurose führt, auch wenn es sich konzeptuell noch nicht um ausgearbeitete Ansätze handelt. Freud ist im vorliegenden Text am ersten topischen Modell des Traums und der Neurose orientiert.

\section{Zur Einbettung von Freuds Abhandlung: Der Wolfsmann}

«Erinnern, Wiederholen und Durcharbeiten» ist 1914 als Teil der behandlungstechnischen Schriften entstanden, die zwischen 1911 und 1915 publiziert wurden. Parallel dazu führte Freud die Analyse des «Wolfsmannes» durch. Über den «Wolfsmann» steht in der editorischen Einleitung der Studienausgabe zu lesen, es handle sich um die «ausführlichste und zweifellos wichtigste aller Krankengeschichten Freuds» (1918b [1914], S. 127), was auch die umfassende Literatur bezeugt, die es über den «Wolfsmann» gibt. Die Analyse des «Wolfsmannes» dauerte von 1910 bis 1914, eine für die damaligen Verhältnisse lange Zeit. 1919 kehrte der Patient zu Freud zurück, es folgten weitere Behandlungen bei weiteren Analytiker_ innen, das Leben des «Wolfsmannes» blieb schwierig. André Green (1999) schreibt über diesen «Grenzfall avant la lettre», er stelle «das schönste [sic!] Scheitern der Psychoanalyse» dar (S. 36; Übers. UG).

Aus demselben Jahr stammt der Text «Über fausse reconnaissance» (1914a), in der Freud anhand kurzer Vignetten dem Phänomen des «déja-vu» nachgeht. Auch hier nimmt er auf den «Wolfsmann» Bezug, der erinnert, wie er als Bub mit einem Messer an einer Baumrinde herum schnitzte und meinte, sich den Finger abgeschnitten zu haben, was sich aber als Halluzination herausstellte. Freud deutet dies im Sinne der Kastrationsangst, die sich in einer Verschiebung vom Penis auf den Finger äussert (S. 119 ff.). Green hält dagegen fest, es handle sich um die 
Halluzination eines Schnittes und Mangels dort, wo gar nichts fehlt: eine Grenzdarstellung der negativen Halluzination (zit. nach Duparc, 2014).

Ebenfalls im Jahr 1914 publizierte Freud «Zur Einführung des Narzissmus», in der er nach der Kontroverse mit Jung und Adler seine Gedanken zum Narzissmus ausführt. In Abgrenzung zu Jung hält Freud daran fest, den Narzissmus mit der Libidotheorie in Verbindung zu bringen und unterscheidet zwischen der narzisstischen Ich-Libido und der Objekt-Libido. Er beschreibt den primären Narzissmus als «Urzustand» des Kindes, in dem die Ich- und Objektlibido noch ungetrennt sind (1914c, S. 167) und führt das Ich-Ideal ein, die Instanz der Selbstbeobachtung, die das Erbe des kindlichen Narzissmus antritt und die Grundlage für die Entwicklung des Über-Ichs bildet.

Stellt man «Erinnern, Wiederholen und Durcharbeiten» in den Kontext dieser Arbeiten, zeigt sich, dass Freud - es ist anzunehmen, im Zuge der Behandlung des «Wolfsmannes» - mit Fragen beschäftigt war, die dem Spektrum des frühkindlichen Narzissmus und der frühen Störungen angehören. Obwohl sich Freud auf das Modell der Neurose konzentriert, ist diese andere Ebene enthalten und liest sich wie ein Subtext, dem ich im Weiteren nachgehen möchte.

\section{Freuds Modell des Traums und der Neurose}

Freud beginnt seine Abhandlung mit einem Rückblick über die Entwicklung der psychoanalytischen Methode, die aus der Hypnose und der Breuer'schen Katharsis entstanden ist. Nach der Hypnose wurde die «heutige» Technik entwickelt, so Freud, die die «Deutungskunst» verwendet, um von der psychischen Oberfläche her die Widerstände bewusst zu machen. Das Ziel bleibt unverändert: «Deskriptiv: die Ausfüllung der Lücken der Erinnerung, dynamisch: die Überwindung der Verdrängungswiderstände» (S. 127) ${ }^{1}$.

Im folgenden Abschnitt beschäftigt sich Freud mit dem Vergessen, das sich dem Erinnern entgegenstellt. Er hält fest, dass das Vergessen auf eine «Absperrung» zurückgehe, die «Verdrängungsschranke», die zwischen bewussten und unbewussten Inhalten trennt und durch Deutung aufgehoben werden könne. Es handle sich um Inhalte, die der Patient, nachträglich gesehen immer schon gewusst, nur nicht bedacht habe - also um symbolisch repräsentierte Inhalte, wie aus heutiger Perspektive gesagt werden kann, wo es Sprache, Sach- und Wortvorstellungen gibt.

Auch die Kindheitsamnesie tritt als Hindernis gegen das Erinnern auf, schreibt Freud, sie werde jedoch durch die Deckerinnerungen «vollständig aufgewogen» (S. 128). Er nimmt an, dass die Deckerinnerungen «die vergessenen Kinderjahre so zureichend (repräsentieren), wie der manifeste Trauminhalt die 
Traumgedanken» (S. 128). Die Kindheitsamnesie lässt sich überwinden, indem das Material aus den Deckerinnerungen entwickelt wird. Freud bezieht sich auf den Traum als Modell. So wie die unbewussten Phantasien und Triebwünsche durch die Traumarbeit entstellt und unkenntlich gemacht werden, um die Zensur zu umgehen und in Form des manifesten Trauminhaltes ins Bewusstsein zu gelangen, so können umgekehrt der manifeste Trauminhalt mit Hilfe der Assoziationen des Träumers erschlossen und die verdrängten Triebwünsche aufgedeckt und gedeutet werden.

Es ist dieses Vorgehen, das Freud in seiner Abhandlung über den «Wolfsmann» anwendet, wo er in akribischer Arbeit den Symptomen, Träumen und Erinnerungen nachgeht und sie minutiös entziffert. Im Einklang mit seinen Annahmen über den Ödipuskomplex deutet Freud die Symptome des Wolfsmannes als Ausdruck der Kastrationsangst und der negativ ödipalen, also homosexuellen Wünschen gegenüber dem Vater.

Neben den äusseren Eindrücken und Ereignissen, die vergessen werden können, erwähnt Freud als zweites die innerpsychischen Vorgänge, wie «Phantasien, Beziehungsvorgänge, Gefühlsregungen und Zusammenhänge», von denen er festhält, dass sie nicht erinnerbar sind, weil sie nie bewusst waren und deshalb auch nicht verdrängt werden können. Anstelle der Verdrängung seien Mechanismen am Werk wie sie bei der Zwangsneurose auftreten, bei der die «Auflösung von Zusammenhängen, Verkennung von Abfolgen, Isolierung von Erinnerungen» im Zentrum stehen (S. 128f.).

Nochmals fühlt man sich an den «Wolfsmann» erinnert, den Freud als Fall von Zwangsneurose behandelt hat, und der bereits als kleiner Bub unter schweren Ängsten, Zwängen und Phobien litt. Doch die Formulierung über die «Auflösung von Zusammenhängen, Verkennung von Abfolgen, Isolierung von Erinnerungen» lässt aus heutiger Perspektive auch an Autoren wie Bion (1967) und dessen Konzept der «Angriffe auf Verbindung» oder an die Arbeit von Green (2002b) über «Die zentrale phobische Position» denken. Green beschreibt darin ein grundsätzlich phobisches Funktionieren des psychischen Apparates, das sich auf das Zerstören von Zusammenhängen ausrichtet, um einem traumatischen Zusammenbruch des Ichs zu entgehen. Es handelt sich um den Bereich der Grenzfälle und der frühen Störungen, wo es nicht primär um unbewusste Konflikte geht, sondern der psychische Apparat insgesamt bedroht ist und sich gegen die Gefahr eines Zusammenbruchs organisiert. Freud fügt eine dritte Bemerkung zum Vergessen an: 
Für eine besondere Art von überaus wichtigen Erlebnissen, die in sehr frühe Zeiten der Kindheit fallen und seinerzeit ohne Verständnis erlebt worden sind, nachträglich aber Verständnis und Deutung gefunden haben, lässt sich eine Erinnerung meist nicht erwecken. Man gelangt durch Träume zu ihrer Kenntnis und wird durch die zwingendsten Motive aus dem Gefüge der Neurose genötigt, an sie zu glauben[.] (S. 129; Hervorh. i. O.)

Freud weist hier den frühen Erfahrungen eine zentrale Bedeutung zu. Er hält fest, dass sie «ohne Verständnis» erlebt werden. Winnicott (1974) nimmt diesen Gedanken in seiner berühmten Arbeit «Die Angst vor dem Zusammenbruch» auf, die weiter unten nochmals zur Sprache kommt. Das kleine Kind - so wäre zu ergänzen - kann sich noch nicht erinnern und vorstellen, was ihm geschieht, weil sein Ich noch nicht genügend entwickelt ist. Frühe Erfahrungen können nur erschlossen und vermutet werden und wir sind darauf angewiesen, uns anhand des Materials der Träume, der Deckerinnerungen oder eben jener «zwingendsten Motive aus dem Gefüge der Neurose» (1914g, S. 129) ein Bild zu machen.

\section{Der Traum der Wölfe}

Wie in der Studienausgabe des Freud-Textes jedoch als Fussnote ${ }^{2}$ (1914g, S. 209) ausgeführt wird, hat Freud bei dieser Bemerkung über die frühen Erlebnisse den «Wolfsmann» im Auge. Im Kapitel IV seiner Abhandlung über den «Wolfsmann» analysiert Freud einen Traum, den dieser als «drei-, vier- oder fünfjähriger Bub» geträumt hat (1918b, S. 54 ff.). Es handelt sich um den berühmten Traum der Wölfe, die vor dem Fenster auf einem Baum sitzen - auf demselben Baum übrigens, der bei der Halluzination des abgeschnittenen Fingers eine Rolle spielt. Die Wölfe sitzen ganz ruhig da, nur das Fenster öffnet sich plötzlich, und der Träumer erwacht in grosser Angst. Es ist dieser Traum, der dem «Wolfsmann» seinen Namen gab. Freud deutet ihn als Darstellung des sexuellen Aktes der Eltern. Er vermutet, der kleine «Wolfsmann» habe als Baby im Zimmer der Eltern schlafend erlebt, wie diese sexuell miteinander verkehrten und sei darüber erwacht. Dieses nicht erinnerbare Erlebnis habe er im Traum der Wölfe nachträglich zur Darstellung gebracht. Hinter der «eigentümlichen Ruhe» des Traumes deutet Freud, in ihr Gegenteil verkehrt, die heftige Bewegung des sich liebenden Paares. Die Zensur ist durch die Ruhe des manifesten Traums getäuscht, wenn nur das Fenster sich nicht öffnen würde. Die Abwehr versagt, wie die Angst des Träumers anzeigt, der über die hereinbrechenden Inhalte erwacht. Ein Angsttraum also. Erstaunlich nur, 
dass Freud nicht die Phantasie der Urszene deutet, sondern auf das reale Ereignis stösst - ein Konkretismus? Eine Ahnung über die fehlende Repräsentanz? Ein traumatisches Element?

Aber Freuds Formulierung: «Man (...) wird durch die zwingendsten Motive aus dem Gefüge der Neurose genötigt, an sie [die frühen Erfahrungen; UG] zu glauben» (1914g, S. 129) weist noch in eine andere Richtung. Zunächst seien Freuds Sprachkunst und analytische Präzision vermerkt: Es drängt sich uns eben auf, wir werden "genötigt», bestimmte Dinge zu denken und «zu glauben», wenn wir uns dem Fluss der Assoziationen überlassen und dem zuwenden, was vom Patienten an Gesagtem und Ungesagtem kommt. Freud bezieht sich hier auf die gleichschwebende Aufmerksamkeit, auf das «Hören mit dem dritten Ohr», wie Reik (1976) diese genuin psychoanalytische Haltung des (Zu-)Hörens genannt hat. Sie bildet mit der freien Assoziation ein Paar und zielt darauf, dem Unbewussten des Patienten das eigene Unbewusste als «empfangendes Organ» zur Verfügung zu stellen (Freud, 1912e, S. 381). Green (2002a) bezeichnet freie Assoziation und gleichschwebende Aufmerksamkeit als «dialogisches Paar» und spricht in Verbindung mit dem Setting vom «(...) Bijou, das im Schmuckkästchen (dem Rahmen) enthalten ist» (S. 55; Übers. UG).

\section{Die Entstehung des psychischen Raums}

In seiner Arbeit über die zentrale phobische Position skizziert Green (2002b) ein Modell der freien Assoziation. Er beschreibt das dichte Netz von Bezügen, Rückbezügen, die vor- und rückwärts gewandte Bewegung des Denkens, das der assoziativen Rede folgt. Es ist eine Rede der «rückwirkenden Rückstrahlung» und «vorausweisenden Ankündigung», der unausgesprochenen Möglichkeiten und retrospektiven Echos (S. 418). Diese «assoziative Ausstrahlung» der Rede verweist auf eine «baumartig verzweigte Struktur des Sinns» (S. 418), der sich ergibt, wenn Analytiker und Analysand miteinander im Kontakt stehen und die Kommunikation von Unbewusst zu Unbewusst gelingt.

Doch dieses Zusammenspiel ist fragil. Green beschreibt Patient_innen, deren psychischer Apparat anti-assoziativ und im Zentrum phobisch eingerichtet ist. Dann spielen die Gedanken nicht. Das Denken wird angegriffen, das Entstehen von Bedeutung verhindert: «(...) der Sinn als Ergebnis der freien Assoziation in der Übertragungsbeziehung (wird) zum Gegenstand einer quasi systematischen Störung und Erstickung», schreibt Green (S. 422; Hervorh. i. O.). Diese phobische Position der Assoziationsvermeidung entspricht Bions Konzept der Angriffe auf 
Verbindung und hat mit der Verwerfung zu tun. Wieder erscheint das «Jenseits» der Neurose.

Auch Roussillon knüpft im Artikel «La pulsion et l'intersubjectivité: vers l'entre-je(u)» (2004) hier an. Das gelingende Zusammenspiel des analytischen Paares, das ebenso ein entre-je, ein «Zwischen-zwei-Ich» wie ein entre-jeu, ein Zusammen-Spiel oder Zwischen-Spiel ist, beruht darauf, dass sich zwei Subjekte begegnen, die ganz und getrennt sind und über einen psychischen Vorstellungsraum verfügen, der durch den Prozess der Subjektivierung entstanden ist, in dem sich die Triebe im Austausch mit dem Objekt haben binden und aneignen lassen und sich das Subjekt entlang der Formel Freuds: «Wo Es war, soll Ich werden» entwickeln konnte (1933a, S. 86).

\section{«Innen Zwischen Aussen»: Wie sich Innen, Aussen, Subjekt und Objekt} aus dem entre-je(u), dem Zwischen- und Zusammenspiel differenzieren

Freuds Überlegungen zum Vergessen und Erinnern bzw. Nicht-ErinnernKönnen weisen also auf die grundsätzliche Frage nach den Bedingungen für die Aneignung von Erfahrung hin. Es geht um die Entstehung des psychischen Raums, um die Entstehung von Vorstellungen und Repräsentanzen und um die Integration der Triebe. Wieder zeigt sich eine Schnittstelle, die über die Neurose hinausweist. Denn die Neurose beruht auf einem Modell, das den psychischen Apparat und seine Funktionen voraussetzt. Im ersten topischen Modell geht Freud davon aus, dass die Triebwünsche und Phantasien im Unbewussten repräsentiert sind und durch das Lust-/Unlustprinzip organisiert werden. Die Triebwünsche können zwar verdrängt, verschoben und verdichtet werden, aber sie bleiben im Unbewussten erhalten und können im Sinne des Lustprinzips und der halluzinatorischen Wunscherfüllung erfüllt werden.

Nach Freud haben sich verschiedene Autor_innen mit der frühen Entwicklung befasst: Melanie Klein als zentrale, frühe Pionierin, Winnicott und Bion, die von ihr ausgehend weitere entscheidende Anstösse gegeben haben, letzterer mit dem Modell des container-contained und den Alpha- und Beta-Elementen. Die Funktion des Primärobjekts wird von Bion so verstanden, dass die Mutter die rohen Erfahrungen und Äusserungsformen des Babys, die Beta-Elemente, aufnimmt und durch ihr eigenes psychisches Funktionieren so verarbeitet, dass sie sie dem Kind auf verdaute und psychisierte, mit Bedeutung verbundene Weise zurückgeben kann (Alpha-Element). Zum Beispiel indem die Mutter das Baby, wenn es schreit, auf den Arm nimmt, es wiegt, mit ihm spricht oder eine Melodie singt, bis es sich 
beruhigt und so die Erfahrung macht, dass es auch psychisch «gestillt» wird und seine Angst aufgenommen und verstanden - das heisst mit Sinn versehen wird.

Green (1966/67) beschreibt die Entwicklung des psychischen Raums im Zusammenhang mit der Trennung vom Primärobjekt. Mit Freud geht er davon aus, dass sich das Objekt in der Abwesenheit konstituiert. In der Abwesenheit beginnt sich das Kind die Mutter über die halluzinatorische Wunscherfüllung vorzustellen und mit Hilfe der inneren Gedächtnisspur die Repräsentanz der Mutter bzw. der Brust als erstem Partialobjekt und Symbol für die Mutter zu bilden. Bion (1962) hat dies im berühmten Satz gefasst: «Wenn da kein «Ding» ist, ist «kein Ding» [no thing/nothing] ein Gedanke» (S. 81 f.). Die genügend gute Anwesenheit der Mutter, das Gehalten-, Versorgt-, Besetzt- und Gespiegelt-Werden bildet die Grundlage für diesen Prozess.

Green nimmt an, dass die Mutter bei der Trennung durch die negative Halluzination ausgelöscht und gleichzeitig als structure encadrante, als rahmengebende Struktur innerlich eingesetzt wird, sodass sich an ihrer Stelle der Raum bildet, der für die Verankerung der Vorstellungen zur Verfügung steht. An Stelle der Verschmelzung und Einheit mit der Mutter entstehen auf diese Weise das (getrennte) Subjekt und der Raum für die Vorstellungen und die Besetzung neuer Objekte. Green (1966/67) schreibt:

\section{Die Mutter wird in den leeren Rahmen der negativen Halluzination hineingenommen und wird so zur rahmengebenden Struktur für das Subjekt selbst. Das Subjekt errichtet sich dort, wo der Einsetzung des Objekts der Vorrang vor dessen Besetzung eingeräumt wurde. (S. 134)}

Green geht davon aus, dass die negative Halluzination der Mutter in dem Moment erfolgt, in dem das Kind die Mutter erstmals als ganzes Objekt wahrnimmt. Deshalb wird mit der negativen Halluzination gleichzeitig auch die Trennung von Ich und Nicht-Ich bzw. Ich und äusserem Objekt begründet.

\section{Wenn der «glatte Ablauf» versagt}

Damit komme ich zum zentralen Thema der Abhandlung von Freud, nämlich jener Gruppe von Patient_innen, bei denen der «glatte Ablauf» der freien Assoziation, der Erinnerung und Deutung des Verdrängten nicht gelingt, weil - wie auf Grund des Gesagten angenommen werden kann - die Voraussetzungen für die Bildung dieses psychischen Innenraums, in dem die Erfahrungen repräsentiert 
sind, nicht gegeben sind. Stattdessen bilden sich «fueros»: abgetrennte psychische Bereiche, wie sie Freud (1896) in seinem berühmten Brief an Fliess genannt hat. Freud (1914g) stellt fest:

So dürfen wir sagen, der Analysierte erinnere überhaupt nichts von dem Vergessenen und Verdrängten, sondern er agiere es. Er reproduziert es nicht als Erinnerung, sondern als Tat, er wiederholt es, ohne natürlich zu wissen, dass er es wiederholt. (S. 129f.)

Zum Beispiel:

Der Analysierte erzählt nicht, er erinnere sich, dass er trotzig und ungläubig gegen die Autorität der Eltern gewesen sei, sondern er benimmt sich in solcher Weise gegen den Arzt. (...) Er erinnert nicht, dass er sich gewisser Sexualbetätigungen intensiv geschämt und ihre Entdeckung gefürchtet hat, sondern er zeigt, dass er sich der Behandlung schämt, der er sich jetzt unterzogen hat, und er sucht diese vor allen geheim zu halten. (S. 129)

Und Freud kommt zum Fazit: «Solange (der Kranke) in Behandlung verbleibt, wird er von diesem Zwange zurWiederholung nicht mehr frei; man versteht endlich, das ist seine Art zu erinnern» (S. 130). Freud führt hier den Wiederholungszwang als Äquivalent für die Erinnerung ein. Durch den Wiederholungszwang gelangt das Material in die Übertragung und wird dadurch erstmals zugänglich. Wiederholen und Agieren dienen also der Darstellung und Kommunikation - wie bei der Patientin, die durch ihre Körperhaltung ein Stück ihrer verlorenen, nichtrepräsentierten Geschichte zum Ausdruck gebracht hatte.

\section{Übertragung, Wiederholung, Widerstand und Agieren}

Freud untersucht nun das Verhältnis von Wiederholungszwang, Übertragung und Widerstand und hält fest, dass «die Übertragung selbst nur ein Stück Wiederholung und die Wiederholung Übertragung der vergessenen Vergangenheit» ist - wobei die Übertragung «nicht nur auf den Arzt, sondern auch auf alle andern Gebiete der gegenwärtigen Situation» falle (S. 130). Freud schreibt: «Je grösser der Widerstand ist, desto ausgiebiger wird das Erinnern durch das Agieren (Wiederholen) ersetzt» (S. 130). Insbesondere, wenn im Verlauf der Behandlung 
die Übertragung feindselig und negativ wird, tritt das Erinnern dem Agieren den Platz ab. Freud:

Von da an bestimmen dann die Widerstände die Reihenfolge des zu Wiederholenden. Der Kranke holt aus dem Arsenal der Vergangenheit die Waffen hervor, mit denen er sich der Fortsetzung der Kur erwehrt und die wir ihm Stück für Stück entwinden müssen. (S. 131)

Welch' kriegerische Metaphern! Zu diesem Kampf um die Entwaffnung lässt sich freilich fragen, ob Freud nicht einer Verführung erlegen ist. Mit Morgenthaler (1978) könnte so argumentiert werden, der dieses «psychische Seilziehen» zwischen Analytiker und Analysand als Agieren der Gegenübertragung und technischen Fehler des Analytikers bezeichnet. Und mit Blick auf den «Wolfsmann» erinnern wir uns an das Zitat über das «schönste Scheitern der Psychoanalyse» (Green, 1999, S. 36), das möglicherweise daraus resultierte. In Freuds Sprache äussern sich aber auch die Schwierigkeiten, die sich in der Arbeit ergeben und wir erfahren, mit welcher Dynamik wir zu rechnen haben, gerade bei frühen Störungen. Wer kennt nicht das Ringen mit der Gegenübertragung, wenn nach vielen Jahren die Patientin immer noch hartnäckig schweigt und Worte nur gegen schwere Hindernisse abzuringen sind?

Kommt die Krankheit in die Übertragung, wiederholt sich «Alles»: «Symptome, Hemmungen, unbrauchbare Einstellungen, pathologische Charakterzüge» (S. 131). Die Krankheit wird zu einem aktuellen Geschehen zwischen Arzt und Patient, wie Freud schreibt:

Wir machen uns klar, (...), dass wir (die) Krankheit nicht als historische Angelegenheit, sondern als eine aktuelle Macht zu behandeln haben. Stück für Stück dieses Krankseins wird nun in den Horizont und in den Wirkungsbereich der Kur gerückt, und während der Kranke es als etwas Reales und Aktuelles erlebt, haben wir daran die therapeutische Arbeit zu leisten, die zum grössten Teil in der Zurückführung auf die Vergangenheit besteht. (S. 131)

Die Schwierigkeiten wiederholen sich also im Hier und Jetzt der Übertragung und sollen in Verbindung mit der Geschichte gedeutet werden. Freud stellt jedoch fest, dass das Heraufbeschwören «dieses Stücks realen Lebens» nicht 
harmlos sei. Er kommt auf die negative therapeutische Reaktion zu sprechen, das «Problem der oft unausweichlichen «Verschlimmerung während der Kur»» (S. 132f.). Aber nur wenige Zeilen später plädiert Freud wieder versöhnlich:

\begin{abstract}
Der Kranke muss den Mut erwerben, seine Aufmerksamkeit mit den Erscheinungen seiner Krankheit zu beschäftigen. Die Krankheit selbst darf ihm nichts Verächtliches mehr sein, vielmehr ein würdiger Gegner werden, ein Stück seines Wesens, das sich auf gute Motive stützt, aus dem es Wertvolles für sein späteres Leben zu holen gilt. (...) (Es geht um die) Versöhnung mit dem Verdrängten, welches sich in den Symptomen äussert (und um eine) Toleranz fürs Kranksein. (S. 132)
\end{abstract}

Wieder zeigt sich Freud schwankend: Voller Zuversicht empfiehlt er, man könne die Patienten «leicht (...) trösten, dass dies (...) vorübergehende Verschlechterungen sind und dass man keinen Feind umbringen kann, der abwesend oder nicht nahe genug ist» - wie er dies fast wörtlich auch in der Abhandlung «Zur Dynamik der Übertragung» (1912b) tut-, um umso deutlicher von den Schwierigkeiten zu sprechen, wenn im Verlauf der Behandlung «neue, tieferliegende Triebregungen (...) zurWiederholung gelangen» (1914g, S. 132). Diese Wiederholungen treten auch ausserhalb der Übertragung auf und können «vorübergehende Lebensschädigungen» oder gar eine dauerhafte Beeinträchtigung der Gesundheit mit sich bringen (S. 132f.).

Erneut verlässt Freud mit seinen Hinweisen das Feld der Neurose. Die Übertragung wird negativ, das Agieren nimmt eine destruktivere Dimension an und sprengt den Behandlungsrahmen, es kommt zu einer Gefährdung von Gesundheit und Leben. Dass Freud gerade hier vom «Kampf» mit dem Patienten spricht, erstaunt deshalb nicht (Freud 1914g):

(Der Arzt) richtet sich auf einen beständigen Kampf mit dem Patienten ein, um alle Impulse auf psychischem Gebiete zurück zu halten, welche dieser aufs Motorische lenken möchte, und feiert es als Triumph der Kur, wenn es gelingt, etwas durch die Erinnerungsarbeit zu erledigen, was der Patient durch eine Aktion abführen möchte. (S. 133) 


\section{Von der Bändigung und Bindung durch die Übertragung}

Freud widmet sich im Weiteren der Frage, was gegen den Wiederholungszwang zu tun sei. Seine Empfehlung: die «Bindung durch die Übertragung»:

Das Hauptmittel aber, den Wiederholungszwang des Patienten zu bändigen und ihn zu einem Motiv fürs Erinnern umzuschaffen, liegt in der Handhabung der Übertragung. (...) Wir eröffnen ihm die Übertragung als den Tummelplatz, auf dem ihm gestattet wird, sich in fast völliger Freiheit zu entfalten, und auferlegt ist, uns alles vorzuführen, was sich an pathogenen Trieben im Seelenleben des Analysierten verborgen hat. Wenn der Patient nur soviel Entgegenkommen zeigt, dass er die Existenzbedingungen der Behandlung respektiert, gelingt es uns regelmässig, allen Symptomen der Krankheit eine neue Übertragungsbedeutung zu geben, seine gemeine Neurose durch eine Übertragungsneurose zu ersetzen, von der er durch die therapeutische Arbeit geheilt werden kann. Die Übertragung schafft so ein Zwischenreich zwischen der Krankheit und dem Leben, durch welches sich der Übergang von der ersteren zum letzteren vollzieht. (S. $134 \mathrm{f}$.)

Bemerkenswerterweise siedelt Freud die Übertragung in einem «Zwischenreich» und «Übergang» an, was an Winnicotts Konzepte des Übergangsraums und der Übergangsphänomene denken lässt. Auch der «Tummelplatz», dieses Bild des Spiels, das durchaus wild sein kann, sich aber in einem sicheren Rahmen bewegt, erinnert an Winnicott. Aber es sind auch die Prozesse der Bindung und Entbindung angesprochen, die Green (2001) im Zusammenhang mit der Objektalisierungsund Desobjektalisierungsfunktion und dem Lebens- und Todestrieb beschrieben hat. Das Freud'sche Diktum der «Bindung durch die Übertragung» erscheint in diesem Kontext als Arbeit an der Bindung oder der Verbindung - z. B. wenn wir mit dem Durchbruch aggressiver oder selbstverletzenden Impulsen konfrontiert sind, die sich im Übertragungsgeschehen über die Jahre langsam verstehen und mit Vorstellungen verbinden lassen. Wir arbeiten dann an der Psychisierung und Darstellung von Unrepräsentiertem und damit an Prozessen, die Freud vorausgesetzt hat, als er auf die Deutung ödipaler Konflikte zielte. Bei der Neurose ist der innere Rahmen im Sinne der structure encadrante etabliert. Es gibt einen «Tummelplatz», ein Übergangs- und Zwischenraum, in dem sich spielen lässt. Bei frühen Störung ist das nicht der Fall. 
Gelingt es, den Wiederholungszwang in der Übertragung zu halten, so formuliert Freud zuversichtlich: «Von den Wiederholungsreaktionen, die sich in der Übertragung zeigen, führen dann die bekannten Wege zur Erweckung der Erinnerungen, die sich nach Überwindung der Widerstände wie mühelos einstellen» (1914g, S. 135).

Dennoch mag Freud seinem «Wundermittel» der Übertragung nicht ganz trauen und baut zusätzlich auf handfeste Absprachen: Die Patient_innen werden verpflichtet, während der Kur keine lebenswichtigen Entscheidungen zu treffen, sondern das Ende der Behandlung abzuwarten. Man muss sich vergegenwärtigen, dass zu Freuds Zeit die Behandlungsdauer wesentlich kürzer und ein solcher Aufschub weniger einschneidend war. Aber wieder sind wir darauf hingewiesen, mit welchen Kräften wir zu tun bekommen und wie schwierig es ist, ihnen einen Rahmen zur Verfügung zu stellen, der das Agieren in Grenzen zu halten vermag. Freud räumt denn auch ein, dass es Fälle gibt, in denen «der Patient in einer Wiederholungsaktion das Band zerreisst» oder die Zeit fehlt, "den wilden Trieben die Zügel der Übertragung anzulegen», wie bei dieser älteren Dame, die in Dämmerzuständen Mann und Haus verlässt und mit einer «gut ausgebildeten, zärtlichen Übertragung» zu Freud kommt, diese jedoch «in unheimlich rascher Weise» steigert, so dass sie auch ihn in kürzester Zeit verlassen hat, noch bevor er etwas hätte unternehmen können, um diese Wiederholung zu stoppen (S. 134). Das Agieren nimmt also auch hier eine Form an, die in Sinne des containment nicht zu halten ist.

Soweit zu Freuds Text. In Bezug auf meine Hypothese lässt sich zusammenfassen, dass Freud eine Gruppe von Patient_innen beschreibt, bei denen der «glatte Ablauf» der freien Assoziation, der Erinnerung und Deutung des Verdrängten scheitert. Das Agieren und Wiederholen von nicht-repräsentierten Erfahrungen steht im Vordergrund und sprengt den Behandlungsrahmen. Die destruktiven Kräfte lassen sich in der Übertragung nicht binden, es kommt zu Abbrüchen und existentieller Gefährdung, wie wir das von Grenzfällen und frühen Störungen kennen.

\section{«Jenseits des Lustprinzips»: Die Grenzfälle}

Green (2012) schreibt, dass Freud mit der Einführung des Wiederholungszwangs ein Prinzip des psychischen Funktionierens entwickelt hat, das für die nicht-neurotischen Störungen paradigmatisch sei. Der psychische Apparat ist nicht wie bei der Neurose um die Wunscherfüllung und das Primat von Lust und Unlust organisiert, sondern im Zentrum stehen die Wiederholung, das Agieren und die Triebabfuhr, welche auf die Entleerung, Evakuierung oder Exkorporation 
von psychischen Inhalten zielt. Es geht um traumatische Inhalte, die in Form von ungebundenen Impulsen und Erregungen durchbrechen. Sie bleiben dem Primärprozess unterworfen, die Verbindung mit der Vorstellung gelingt wegen des traumatischen Gehaltes nicht. Die Prozesse der Vorstellung und Symbolisierung werden durch das Agieren kurzgeschlossen und die gefährlichen Inhalte evakuiert.

Mit Donnet hat Green im Buch L'enfant de ça (1973) das Konzept der «weissen Psychose» entwickelt, die er als «Keim der Psychose» bezeichnet. Sie äussert sich vor allem in einem Angriff auf den Denkapparat, ohne dass es zu einer manifesten Psychose kommt. Das Buch beruht auf der Analyse eines Erstgespräches mit einem jungen Mann, der von sich sagte, er sei l'enfant de ça, womit er den Inzest seiner Mutter mit ihrem Cousin meint, aus dem er gezeugt wurde - wissend/nichtwissend, dass er damit auf das Es (le ça) abzielte.

Die Merkmale der weissen Psychose lassen sich nach Duparc (2014) wie folgt zusammenfassen:

1. Ein schlecht strukturierter Ödipus mit Merkmalen einer Bi-Triangulierung (Green), in dem Mutter und Vater schlecht differenziert und entlang einer Spaltung organisiert sind, so dass sie jeweils das Negativ des Andern darstellen, ohne dass es einen wirklichen Dritten gibt.

2. Eine Störung des Narzissmus und der Grenzen zwischen Subjekt und Objekt, so dass das Subjekt gefangen ist zwischen der Angst vor der Intrusion des übermässig anwesenden Objekts und der Angst vor dem VerlassenWerden durch das übermässig abwesende Objekt. Zwischen Intrusion und Trennungsangst, zwischen Übererregung und Leere.

3. Der Triumph des Todestriebes, welcher sich auf das Denken und die Vorstellungen der Objekte und des eigenen Körpers auswirkt, so dass das Agieren und Entleeren oder Evakuieren oder Exkorporieren von psychischen Prozessen im Vordergrund steht.

Green und Donnet erläutern Merkmale einer Borderline-Störung. Die Ängste, die dabei eine Rolle spielen, sind mit der Kastrationsangst, die Freud beim «Wolfsmann» gedeutet hat, nicht vergleichbar. Es geht um archaische Ängste, wie Melanie Klein, Winnicott, Bion und andere sie beschrieben haben: namenlose Angst, Vernichtungsangst, katastrophische Angst.

Winnicott (1990 [1974]) geht im bereits erwähnten Artikel «Die Angst vor dem Zusammenbruch» davon aus, dass die «undenkbaren Seelenqualen» in der Übertragung als Angst vor einem Zusammenbruch imponieren. Er nimmt an, dass es sich dabei um die Wiederholung eines Zusammenbruches handelt, der in der 
Vergangenheit bereits stattgefunden hat und zwar als Folge eines Versagens der primären Umgebung. Dieser Zusammenbruch wurde erlitten, aber nicht erlebt, weil das Ich nicht genügend reif war, um das Geschehen als eigene Erfahrung unter Kontrolle zu bringen (S. 1120f.) - wie das in Freuds oben markierter Formulierung angeklungen ist. Winnicott schreibt:

Der Patient muss sich daran «erinnern», aber es ist unmöglich, sich an etwas zu erinnern, das noch nicht geschehen ist, und dieses vergangene Geschehen ist noch nicht passiert, weil der Patient noch nicht (wirklich) da war (...). In diesem Fall ist der einzige Weg für den Patienten, sich zu «erinnern», der, das Vergangene erstmals in der Gegenwart, d.h. in der Übertragung, zu erleben. Das Vergangene und Zukünftige wird so zu einem Gegenstand des Hier und Jetzt und zum ersten Mal vom Patienten erlebt. Ein Äquivalent zur Erinnerung, und im Resultat ein Äquivalent zur Aufhebung der Verdrängung, wie wir es aus der Analyse psychoneurotischer Patienten (...) kennen. (S. 1122)

Winnicott bezieht sich unverkennbar auf Freuds Text. Er betont jedoch einen Aspekt der Wiederholung, der eine Zwischenstellung einnimmt. Zwar handelt es sich um traumatische Ängste, aber die Wiederholung in der Übertragung ermöglicht ein Erleben im Dienste des Ichs. Zum Schluss möchte ich deshalb noch einen anderen Bogen schlagen.

Freud beschreibt in «Jenseits des Lustprinzips» (1920g) im Abschnitt über das Fadenspulenspiel seinen kleinen Enkel. Dieser spielt mit einer Fadenspule, die er von sich wegwirft, was er stimmlich mit einem langgezogenen «o-o-o-o» begleitet, um sie dann wieder zu sich zurückzuziehen und mit freudigem «Da» zu begrüssen (S. 12f.). Freuds Deutung dieses Spiels: Der kleine Enkel wiederholt die Erfahrung mit seiner Mutter, die ihn verlässt und wieder zurückkehrt. Unter Wendung von passiv in aktiv bringt er ein Ereignis unter seine Kontrolle, dem er ausgeliefert war und stellt es im Spiel symbolisch dar. In Bezug auf den referierten Freud-Text liesse sich formulieren: Der kleine Enkel erinnert und wiederholt, was er erlitten hat und kann diese Erfahrung in seinem Spiel durcharbeiten, immer wieder, um sie unter seine Kontrolle zu bringen, sich ihrer zu bemächtigen und sie sich anzueignen.

In dieser von Winnicott ausgehenden Perspektive erscheint «Erinnern, Wiederholen und Durcharbeiten» als Prinzip der Aneignung und Integration von 
Erfahrung, das der Subjektwerdung dient. Die Wiederholung steht im Dienste des Ichs und hat eine konstruktive, der Bewältigung dienende Funktion. Im Gegensatz dazu kann die Wiederholung mit den destruktiven Kräften in Verbindung stehen, die das Ich bedrohen und zum Zusammenbruch führen. Gelingt es, die Wiederholung im Rahmen der analytischen oder therapeutischen Situation mit dem Patienten zu halten und zu binden, ohne dass wir zerstört werden, kann sich die nicht repräsentierte Erfahrung transformieren und erträglicher werden. Aber wie schon Freud wusste: Es braucht Zeit und gelingt nicht immer.

\section{Anmerkungen}

1 Sämtliche Zitate stammen, wo nicht anders vermerkt, aus dem referierten Freud-Text von 1914.

2 Die Fussnote lautet: «Der Hinweis [über die frühen Kindheitserinnerungen; UG] bezieht sich auf den ‘Wolfsmann und seinen Traum aus dem fünften Lebensjahr. Freud hatte diese Analyse gerade abgeschlossen und war möglicherweise dabei, etwa gleichzeitig mit der vorliegenden Arbeit, die Krankengeschichte niederzuschreiben, obgleich diese dann erst vier Jahre später veröffentlicht wurde (1918b) (...).» (S. 209 der Studienausgabe zu Freud, $1914 \mathrm{~g})$.

\section{Literatur}

André, J. (1999). Les états limites. PUF.

Bion, W. R. (2013 [1967]). Frühe Vorträge und Schriften mit einem kritischen Kommentar: «Second thoughts». Brandes \& Apsel.

Bion, W. R. (1992 [1962]). Lernen durch Erfahrung. Suhrkamp.

Donnet, J.-L. \& Green, A. (1973). L'enfant de ça. Psychanalyse d'un entretien: La psychose blanche. Les éditions de minuit.

Duparc, F. (2014). De l'hallucination négative à la psychose blanche. Vortrag am Freud-Institut Zürich, Schweiz.

Freud, S. (1896). Aus den Anfängen der Psychoanalyse. Briefe an Wilhelm Fliess. Fischer.

Freud, S. (1912b). Zur Dynamik der Übertragung. GWVIII, 364-374.

Freud, S. (1912e). Ratschläge für den Arzt bei der psychoanalytischen Behandlung. GWVIII, 376-387.

Freud, S. (1914a). Über fausse reconnaissance («déjà-racconté») während der psychoanalytischen Arbeit. GWX, 116-123.

Freud, S. (1914c). Zur Einführung des Narzissmus. GWX, 137-170.

Freud, S. (1914g). Weitere Ratschläge zur Technik der Psychoanalyse: II. Erinnern, Wiederholen und Durcharbeiten. GWX, 126-136. 
Freud, S. (1918b [1914]). Aus der Geschichte einer infantilen Neurose (Der «Wolfsmann»). GWXII, 27-157.

Freud, S. (1920g). Jenseits des Lustprinzips. GW XIII, 1-69.

Freud, S. (1933a). Neue Folge der Vorlesungen zur Einführung in die Psychoanalyse. GWXV, 62-86.

Green, A. (2012). La Clinique psychanalytique contemporaine. Ithaque.

Green, A. (2002a). Idées directrices. Pour une psychanalyse contemporaire. PUF.

Green, A. (2002b [2000]). Die zentrale phobische Position - mit einem Modell der freien Assoziation, Psyche, 56(5), 409-441.

Green, A. (2001). Todestrieb, negativer Narzißmus, Desobjektalisierungsfunktion. Psyche, 55(09-10), 869-877.

Green, A. (1976). Einer, Anderer, Neutrum: Wertskalen desselben. In ders. (2004), Die tote Mutter. Psychoanalytische Studien zu Lebensnarzissmus und Todesnarzissmus. (S. 31-86). Psychosozial-Verlag.

Green, A. (1966/ 1967). Primärer Narzissmus: Struktur oder Zustand. In ders. (2004), Die tote Mutter. Psychoanalytische Studien zu Lebensnarzissmus und Todesnarzissmus. (S. 87-140. Psychosozial-Verlag.

Morgenthaler, F. (1986). Technik. Zur Dialektik der psychoanalytischen Praxis. Syndikat

Pirlot, G. \& Cupa, D. (2012). André Green. Les grands concepts psychanalytiques. PUF. Reik, Th. (1976). Hören mit dem dritten Ohr. Die innere Erfahrung eines Psychoanalytikers. Hoffmann \& Campe.

Roussillon, R. (2004). La pulsion et l'intersubjectivité: vers l'entre-je(u). In ders. (2008), Le jeu et l'entre-je(u). (S. 1-22). PUF.

Winnicott, D. W. (1974). Die Angst vor dem Zusammenbruch. Psyche, 45(12), 1116-1126.

\section{Angaben zur Autorin}

Uorschla Guidon ist 1967 in Scuol geboren. Sie studierte an der Universität Zürich Psychologie, Psychopathologie und Sozialpädagogik und bildete sich am PSZ zur Psychotherapeutin und Psychoanalytikerin aus. Sie arbeitete von 2000-2001 am Psychiatrischen Ambulatorium in Luzern, von 2001-2010 als Einzeltherapeutin und Leiterin Psychotherapie im Therapieheim Sonnenblick in Kastanienbaum, einem Heim für weibliche Jugendliche mit schweren Entwicklungskrisen. Von 2010-2018 war Uorschla Guidon in der Seminarleitung, in der Weiterbildungskommission und in der Akkreditierungsgruppe des PSZ engagiert. Sie ist Dozentin für die 
Weiterbildung, lebt in Zürich und arbeitet in einer Praxisgemeinschaft als Psychotherapeutin, Psychoanalytikerin und Supervisorin. 